\title{
Cost efficiency in the detection of chytridiomycosis using PCR assay
}

\author{
Kerry M. Kriger ${ }^{1,2, *}$, Jean-Marc Hero ${ }^{1}$, Kevin J. Ashton ${ }^{3}$ \\ ${ }^{1}$ Griffith University, Centre for Innovative Conservation Strategies, PMB 50 Gold Coast Mail Centre, Queensland 9726, Australia \\ ${ }^{2}$ Save The Frogs, Centre for Advanced Scientific Research, 10995 Poplar Ford Trail, Manassas, Virginia 20109, USA \\ ${ }^{3}$ Griffith University, Heart Foundation Research Centre, PMB 50 Gold Coast Mail Centre, Queensland 9726, Australia
}

\begin{abstract}
Chytridiomycosis is an emerging infectious disease of amphibians associated with mass mortalities and population declines worldwide. Recent technological advances have resulted in a highly sensitive, non-invasive technique for diagnosing the disease based on a quantitative (realtime) polymerase chain reaction (qPCR) assay. The qPCR assay yields the most accurate and informative data of any available detection technique. However, due to the relatively high costs involved, it has yet to attain widespread use by chytridiomycosis researchers. Using the results of a disease survey of 467 wild frogs from eastern Queensland, Australia, we examine the necessity of triplicate assays in qPCR detection of chytridiomycosis. We describe a singlicate qPCR assay that can be used to substantially decrease costs, with no significant decrease in sensitivity. We also demonstrate that detection of chytridiomycosis by use of the conventional PCR assay may lead to appreciable underestimations in disease prevalence. We recommend that amphibian disease researchers adopt the singlicate qPCR assay as the primary means of chytridiomycosis detection.
\end{abstract}

KEY WORDS: Batrachochytrium dendrobatidis $\cdot$ Amphibian declines $\cdot$ Chytridiomycosis $\cdot$ Real-time Taqman PCR assay · Chytrid

\section{INTRODUCTION}

A quantitative (real-time) polymerase chain reaction (qPCR) assay has recently been developed for the detection of chytridiomycosis (Boyle et al. 2004), an emerging infectious disease associated with amphibian population declines worldwide (Berger et al. 1998, Lips 1999, Bosch et al. 2001, Green et al. 2002, Ron et al. 2003, Weldon \& du Preez 2004). This assay has been demonstrated to be the most reliable means of detecting the disease in both wild and captive amphibians (Boyle et al. 2004, Kriger et al. 2006, this issue). It is also the only technique available that is capable of accurately quantifying the number of Batrachochytrium dendrobatidis (causative agent of chytridiomycosis) zoospores present on samples, versus yielding solely presence/absence data (as is the case with histology or conventional PCR, the most commonly used alternatives to qPCR). However, qPCR has yet to attain widespread use. This is primarily due to the high costs involved in the laboratory analysis, with prices ranging from US\$13 to $\$ 40$ per sample, depending on whether samples are analyzed at cost in the researcher's own laboratory or if the samples must be outsourced to a commercial laboratory. As many chytridiomycosis studies require hundreds (Berger et al. 2004, Burrowes et al. 2004, Retallick et al. 2004, Beard \& O'Neill 2005, Daszak et al. 2005), thousands (Aplin \& Kirkpatrick 1999, McDonald et al. 2005, Ouellet et al. 2005) or tens of thousands (Speare et al. 2005) of samples, these costs can become prohibitive to most researchers, leading them either to perform studies with sample sizes too small to achieve acceptable statistical power, or to use less expensive methods of analysis (e.g. histology or conventional PCR) that may compromise the quality and/or usefulness of the data (Kriger et al. 2006). As such, progress in chytridiomycosis research will be hindered unless a more economic method of disease detection becomes available. 
The triplicate assay, in which a sample is analyzed 3 times, is the generally accepted method used in qPCR analysis, be it chytridiomycosis (Boyle et al. 2004), biomedical (Donovan et al. 2000, Ginzinger 2002, Emery et al. 2004), or microbiological (Helps et al. 2001, Okano et al. 2004) research. Triplicate assays are thought to reduce the chances of both false positives (i.e. contamination) and false negatives (i.e. missed detection), and thus bring a higher degree of accuracy to the results than if tests were performed in singlicate. However, triplicate analyses require significant increases in chemicals and labor (and associated costs). Using the results of a chytridiomycosis survey of 467 wild frogs from eastern Queensland, Australia, we examine the necessity of triplicate assays in qPCR detection of chytridiomycosis, and describe a method of analysis that can be used to significantly decrease costs, while sacrificing no significant decrease in sensitivity.

Many researchers worldwide are currently using the conventional PCR assay described by Annis et al. (2004) to detect chytridiomycosis. However, the effect that the lowered sensitivity of this assay (relative to that of qPCR) has on the results of disease surveys has never been examined. We used the data from our survey of wild frogs to quantify the number of false negatives (and subsequent underestimation in disease prevalence) we could have expected had we analyzed our samples using the conventional PCR assay.

\section{MATERIALS AND METHODS}

Field collection. Between December 2003 and March 2005, 415 frogs from SE Queensland, Australia (Springbrook, Lamington and Conondale National Parks) and 52 frogs from mid-eastern Queensland (Eungella National Park) were caught using clean, unused plastic bags. We sampled the frogs for chytridiomycosis by firmly running a cotton swab (Medical Wire \& Equipment, MW 100-100; sourced from Biomerieux) 10 times over the frog's dorsal surface, 10 times over each of the frog's sides (from groin to armpit), 10 times over the ventral surface, 10 times on the undersides of each thigh, and 5 times on the underside of each foot. This technique was chosen to maximize the amount of surface area sampled, thus minimizing the likelihood of obtaining false negatives. Swabs were then replaced in their original container, and were frozen at $-20^{\circ} \mathrm{C}$ upon return from the field (within $10 \mathrm{~h}$ of sampling). Swabs remained frozen until qPCR analysis could take place.

Laboratory analysis. Swabs were analyzed in triplicate using established qPCR techniques (Boyle et al. 2004). Changes to the method of Boyle et al. (2004) were as follows: (1) nucleic acids were extracted using $50 \mu$ PrepMan Ultra; (2) the tip of the swab was used instead of a toe; (3) a positive control (swab dipped in a broth of Batrachochytrium dendrobatidis culture) was analyzed on every plate; (4) the negative controls were diethyl procarbonate (DEPC)-treated $\mathrm{H}_{2} \mathrm{O}_{;}$(5) $20 \mu \mathrm{l}$ reactions $(10 \mu \mathrm{l}$ of $2 \times$ Taqman Universal PCR master mix, $5 \mu$ l of diluted [1:10] DNA, $2 \mu$ l DEPC-treated $\mathrm{H}_{2} \mathrm{O}, 900 \mathrm{nM}$ of each primer, and $250 \mathrm{nM}$ Taqman MGB probe) were used, as opposed to the $25 \mu$ reactions described by Boyle et al. (2004), reducing chemical costs by approximately $20 \%$.

Swabs for which Batrachochytrium dendrobatidis was detected in all 3 wells of the triplicate assay were considered positive for chytridiomycosis, and swabs for which $B$. dendrobatidis was not detected in any well were considered negative. Swabs for which $B$. dendrobatidis was detected in 1 or 2 , but not all 3 wells, were initially considered suspicious, and were re-analyzed using the supernatant that remained from the original DNA extraction. Upon re-analysis, suspicious swabs that yielded no positive wells were considered to be negative for chytridiomycosis, the original positive well(s) assumed to be from inadvertent contamination. Suspicious swabs which yielded at least 1 positive well upon re-analysis were considered to be positive for chytridiomycosis, the original discrepancy in results assumed to have arisen not from contamination, but from the inherently high variation possible when amplifying DNA with very low initial copy numbers (Peccoud \& Jacob 1996).

Data analysis. The prevalence of chytridiomycosis in the sample population as determined by the triplicate analyses was calculated by dividing the total number of positive samples by the total number of samples analyzed. By applying the following criteria to the data from our triplicate analyses, we were also able to calculate the most likely results we would have obtained had our samples been analyzed in singlicate: we assumed that any swab which yielded 3 positive wells in the triplicate analysis would have yielded a positive well if it had been analyzed in singlicate, and that swabs yielding no positive wells in the triplicate analysis would not have yielded positive wells had they been analyzed in singlicate. By chance, two-thirds of swabs yielding 2 positive wells in the initial triplicate assay would have been positive in the singlicate assay, and one-third of swabs yielding only 1 positive well in the triplicate assay would have been positive in the singlicate assay. A chi-squared test was used to test for any significant difference in prevalence between the 2 methods.

Sensitivity (the probability that a diagnostic test will yield a positive result if the condition is truly present) and specificity (the probability that a diagnostic test 
will yield a negative result if the condition is truly absent) of the singlicate qPCR assay were determined using the web-based program available at VassarStats (http://faculty.vassar.edu/lowry/VassarStats.html), and by designating the final results of the triplicate qPCR assay as the 'true' disease status of the samples.

In order to quantify the number of false negatives expected had we analyzed our samples using the conventional PCR assay described by Annis et al. (2004), we summed the total number of positive samples that yielded triplicate means of less than 10 Batrachochytrium dendrobatidis zoospores. As the lower detection limit of the conventional PCR assay is about 10 zoospores (Annis et al. 2004), we assumed that the $B$. dendrobatidis on these samples would have gone undetected by a conventional PCR assay.

Estimation of qPCR costs. The cost associated with qPCR varies between laboratories, based on differences in machine use fees, chemical and consumable costs, technician wages, etc. We calculated the cost of qPCR using conservative estimates based on our own experience, and corroborated by other laboratories. In all instances we biased cost estimates toward the lower end of the spectrum.

For the triplicate assay, we assumed that 24 samples would be run per 96-well PCR plate, the remaining wells being used for standards (known quantities of Batrachochytrium dendrobatidis zoospores necessary for accurate quantitation), positive and negative controls. We estimated a flat rate of US\$10 sample $^{-1}$ for chemicals, consumables and machine use fees, and $\$ 3$ sample $^{-1}$ for labor $\left(\$ 20 \mathrm{~h}^{-1} ; 27\right.$ samples can be analyzed in $4 \mathrm{~h}$ : Boyle et al. 2004), resulting in a price of $\$ 13$ sample $^{-1}$. A detailed list of costs and suppliers can be obtained from the primary author (K.M.K.) upon request.

Cost for qPCR using a singlicate assay was calculated by assuming that 93 samples could be analyzed per 96-well PCR plate, with the 3 remaining wells being used for a standard, a negative control, and a positive control. We assumed the researcher would confirm in triplicate any swab that yielded a positive result in the singlicate assay. Re-analysis in triplicate using a full set of standards is necessary in order to accurately quantify the number of Batrachochytrium dendrobatidis zoospores. If none of the 93 samples yield positive results, a triplicate re-analysis is unnecessary. However, increasing numbers of positive results from the singlicate run would result in more triplicate re-analyses being required, and subsequent increases in the amount of chemicals and labor required. Thus the cost of the singlicate assay varies in direct proportion to the prevalence of chytridiomycosis in the population being sampled.

\section{RESULTS}

Of 467 swabs analyzed in triplicate using the qPCR assay, 140 tested positive for chytridiomycosis (Batrachochytrium dendrobatidis was detected in all 3 wells); 14 swabs yielded suspicious results $(B$. dendrobatidis was initially detected in 2 of 3 wells for 8 swabs, and in one of 3 wells for 6 swabs); 313 swabs tested negative. The 14 suspicious swabs were re-analyzed: 8 swabs yielded at least 1 positive well upon re-analysis and were thus considered positive, whereas the other 6 did not yield a positive well upon re-analysis, and were thus deemed negative. There were therefore 148 positive samples and 319 negative samples, giving a total prevalence of $31.7 \%$ using the triplicate qPCR assay (Tables 1 \& 2).

The number of Batrachochytrium dendrobatidis zoospores found on 'suspicious' samples was significantly lower (mean zoospores in positive wells $=4.8, \mathrm{SE}=1.2$, max. $=11$ ) than that found on positive swabs (mean zoospores per well $=3532, \mathrm{SE}=925, \max .=100489$; Mann-Whitney $U$-test: $Z=5.9, \mathrm{p}<0.001$ ) (Fig. 1), and all swabs with more than 11 zoospores detected

Table 1. Prevalence of chytridiomycosis in wild frogs as determined by triplicate quantitative PCR (qPCR) assay, and as predicted for singlicate $\mathrm{qPCR}$ assay and conventional PCR assay. Final triplicate results incorporate results of the reanalysis of the 14 initially suspicious swabs ( $\mathrm{n}=467$ swabs analyzed in triplicate using qPCR). na: not applicable

\begin{tabular}{|lcccc|}
\hline Sample & \multicolumn{2}{c}{ Triplicate } & \multicolumn{2}{c|}{ Predicted results for } \\
& Initial & Final & Singlicate & Conventional PCR \\
\hline Positive & 140 & 148 & 147.33 & 125 \\
Suspicious (2/3) & 8 & na & na & na \\
Suspicious (1/3) & 6 & na & na & na \\
Negative & 313 & 319 & 319.67 & 342 \\
Prevalence (\%) & & 31.7 & 31.5 & 26.8 \\
\hline
\end{tabular}

Table 2. Results of chytridiomycosis survey of 467 wild frogs from eastern Queensland, Australia

\begin{tabular}{|lrc|}
\hline Species & $\mathrm{n}$ & Prevalence (\%) \\
\hline Litoria pearsoniana & 210 & 48.6 \\
Litoria wilcoxii & 118 & 29.7 \\
Litoria chloris & 103 & 8.7 \\
Mixophyes fasciolatus & 11 & 0 \\
Litoria gracilenta & 7 & 0 \\
Litoria latopalmata & 6 & 0 \\
Litoria peronii & 4 & 0 \\
Taudactylus eungellensis & 2 & 50 \\
Mixophyes iteratus & 2 & 0 \\
Adelotus brevis & 1 & 100 \\
Limnodynastes peronii & 1 & 0 \\
Lechriodus fletcheri & 1 & 0 \\
Bufo marinus & 1 & 0 \\
\hline
\end{tabular}




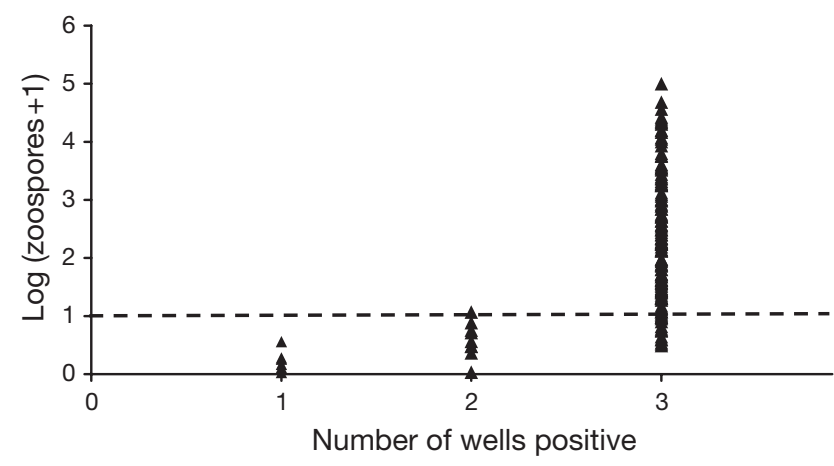

Fig. 1. Batrachochytrium dendrobatidis. Quantitation of zoospores detected on samples $(n=467)$ yielding different results in triplicate qPCR assay (expressed as the triplicate mean). Dashed line represents lower limit of detection of conventional PCR assay (Annis et al. 2004)

yielded positive results for all 3 wells of the triplicate assay. This validates our rationale for labeling positive all initially suspicious samples that yielded at least 1 positive well upon triplicate re-analysis: the high variation expected when amplifying DNA with very low initial copy numbers (Peccoud \& Jacob 1996), and not contamination of a well, was the likely cause of the conflicting well-to-well results.

The theoretical results had our samples been analyzed in singlicate are given in Table 1 . We assumed that 2 of the 6 samples with 1 well positive in the triplicate assay would have been diagnosed positive in a singlicate assay, and that (on average) 5.33 of the 8 samples with 2 wells positive in the triplicate assay would have been diagnosed positive in a singlicate assay. This results in a predicted prevalence of $31.5 \%$ had the samples been analyzed using a singlicate qPCR assay. Thus, the difference in prevalence between the singlicate and triplicate methods was only $0.2 \%$. This difference was not significant $\left(\chi_{1,0.05}^{2}=0.06 ; \mathrm{p}=0.81\right)$. Sensitivity and specificity of the singlicate qPCR assay were high, at 98 and $99 \%$, respectively.

Of 148 positive swabs, $15.5 \%(n=23)$ had triplicate means of $<10$ zoospores (mean $=4.7 ; \mathrm{SE}=0.54$; range $=1.3$ to 9.4) (Fig. 1). The Batrachochytrium dendrobatidis on these 23 swabs would possibly have gone undetected had we analyzed our samples using conventional PCR, and this would have resulted in an assumed disease prevalence of $26.8 \%$, a predicted underestimation of 4.7 and $4.9 \%$ compared to the singlicate and triplicate qPCR assays, respectively (Table 1).

While the cost per sample for swabs analyzed in triplicate remains constant at US\$13, the cost to analyze samples using the singlicate qPCR assay varies from a minimum of $\$ 5.34$ sample $^{-1}$ (0\% prevalence, no triplicate re-analysis required) to $\$ 14.89$ sample $^{-1}(100 \%$ prevalence, all samples requiring triplicate re-analysis). The expected cost of analyzing swabs taken from populations with varying disease prevalence is given in Fig. 2. The monetary savings of the singlicate assay are associated with reductions in chemicals, consumables, machine use fees, and labor (singlicate analyses are less time-consuming).

\section{DISCUSSION}

We have shown that the use of a singlicate assay in qPCR detection of chytridiomycosis can provide significant economic benefits over the use of a triplicate assay, while sacrificing no significant loss of accuracy. For instance, we calculate that in this study we could have saved US\$2163 by using a singlicate assay. Given the high number of chytridiomycosis studies now taking place worldwide, the singlicate analytical approach described herein has significant financial bearing. A maximum saving of $\$ 7.66$ sample $^{-1}$ can be attained by using the singlicate assay when there is no chytridiomycosis detected in the population (Fig. 2). This is a reduction in costs of up to $59 \%$ compared with the triplicate assay, and makes qPCR comparable in cost to other diagnostic techniques in current use.

At any prevalence less than $80.2 \%$, it is more economical to use the singlicate assay (Fig. 2). We could only find 1 disease survey (Hanselmann et al. 2004) in which prevalence of chytridiomycosis in a wild amphibian population was greater than $80.2 \%$. All other published surveys have reported chytridiomycosis prevalence <60\% (Aplin \& Kirkpatrick 1999, Hopkins \& Channing 2003, Retallick et al. 2004, Weldon et al. 2004, Daszak et al. 2005, McDonald et al. 2005, Ouellet et al. 2005, Kriger et al. 2006). The

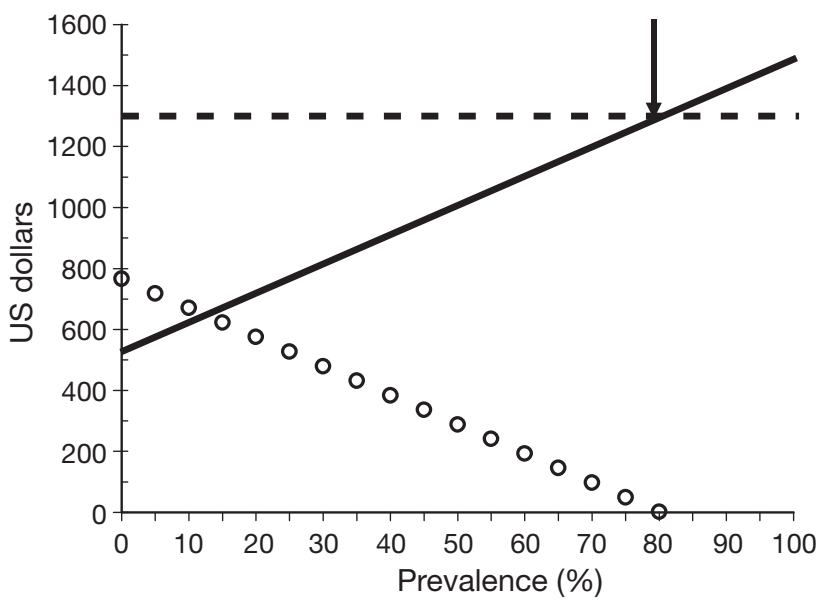

Fig. 2. Costs required to analyze 100 samples for chytridiomycosis using triplicate (dashed line) and singlicate (continuous line; $y=9.55 x+534$ ) qPCR assays, at varying levels of disease prevalence. Amount of money saved by using singlicate assay is also shown (O). Arrow indicates the prevalence (80.2\%) below which it is more cost efficient to perform singlicate assays 
majority of chytridiomycosis studies on wild amphibian populations are therefore likely to benefit from the singlicate qPCR assay described herein. This has important implications for chytridiomycosis research, as it will allow researchers to perform studies with greater sample sizes than were previously possible for a given budget. This will increase their chance of having enough statistical power to detect the trends under investigation, thus yielding more informative data on the ecology of the disease.

The reduced costs involved in the singlicate qPCR assay will also assist those involved in the exportation of amphibians for the zoo, pet or food trades, as it will enable them to increase the number of amphibians surveyed for chytridiomycosis, thereby reducing the chances that they unwittingly transport the disease to naïve amphibian populations.

Laboratory research on chytridiomycosis should also derive economic benefit from the singlicate qPCR assay, although it should be emphasized that the assay used in a given study should always be chosen based on knowledge of the specific population in question. For instance, if a study involves experimental infections or sampling of amphibians during a disease outbreak, a sufficiently high proportion of the population $(>80.2 \%)$ might be infected as to render the triplicate assay potentially more cost effective than the singlicate assay (Fig. 2).

Furthermore, the precise question the researcher is trying to answer will affect not only the decision of which assay is best-suited to a particular study, but also the precise methodology to be utilized. Whereas most field studies are concerned with disease prevalence in populations, the diagnosis of an individual animal's disease status in certain mark-recapture studies, laboratory experiments or captive-breeding programs may have such important ramifications that the extra costs of the triplicate assay may be warranted. Alternatively, a researcher whose sole goal is to determine whether or not chytridiomycosis is present or absent in a population may have no interest in the disease status of individuals, the severity of their infections, or even the prevalence in the population. In such a case, using a singlicate assay and pooling 5 to 10 samples per well might be a highly cost-effective option. A singlicate re-analysis of any positive pools could be used to confirm that the positive result was not due to contamination (triplicate re-analysis with a full set of standards would be unnecessary since quantitation is not required). The efficacy and details of pooling samples should be more thoroughly explored.

Many researchers worldwide are currently utilizing conventional PCR techniques (Annis et al. 2004) for the detection of chytridiomycosis. This is likely due to 2 factors: (1) monetary restrictions, i.e. the conventional
PCR method does not use a triplicate assay, and is therefore less expensive than the previously described (Boyle et al. 2004) qPCR technique. The singlicate qPCR technique described herein, however, is approximately equal in cost to conventional PCR techniques. (2) There is a false perception that the difference in sensitivity between conventional PCR and qPCR is negligible. However, qPCR is widely regarded as being more sensitive than conventional PCR (Luthra et al. 1998, Helps et al. 2001, Borg et al. 2003, Emery et al. 2004). With regard to the detection of Batrachochytrium dendrobatidis, the qPCR method described by Boyle et al. (2004) can detect a single chytrid zoospore, whereas the conventional PCR method described by Annis et al. (2004) sets a lower detection limit of about 10 zoospores, under which reproducibility is poor. In this study, $15.5 \%$ of the positive swabs $(\mathrm{n}=148)$ had less than $10 \mathrm{~B}$. dendrobatidis zoospores. Boyle et al. (2004) and Kriger et al. (2006) also found many lightly infected individuals, with $18.0 \%(\mathrm{n}=61)$ of infected samples in the former study having less than 10 chytrid zoospores, and $40.0 \%(\mathrm{n}=15)$ in the latter. As the $B$. dendrobatidis on these samples might have gone undetected in a conventional PCR assay, we suggest that the difference in sensitivities between conventional PCR and qPCR may be of greater concern than previously realized, and that past studies that have employed the conventional PCR assay may have significantly underestimated the prevalence of chytridiomycosis. It should be noted however, that the comparisons made in this study are theoretical, and that empirically derived data would provide more in-depth information. As a final note in comparing conventional PCR and $\mathrm{qPCR}$, by eliminating the need for postamplification product processing, qPCR both reduces the time required to obtain results and removes one potential avenue for sample contamination (Emery et al. 2004).

The cost of qPCR machines has dropped dramatically in recent years, and many universities now have core facilities that employ skilled technicians to perform qPCR analyses for university researchers, increasing the accessibility to this technology. The sensitivity of qPCR is superior to that of other diagnostic techniques. It is the sole technique capable of quantifying disease levels, and when making use of singlicate assays, it is comparable in price to other available diagnostic techniques. As such, we recommend that amphibian disease researchers adopt qPCR as the primary means of chytridiomycosis detection.

Acknowledgements. We thank the Eppley Foundation for Research, the National Geographic Society's Committee for Research and Exploration, and the Australian Society of Herpetologists for funding this study. We also thank the 
Consortium for Conservation Medicine for financial assistance. K.M.K. received support from the Griffith University School of Environmental \& Applied Sciences. The Terrestrial Ecology Laboratory Group and 2 anonymous reviewers provided helpful comments on an earlier version of the manuscript, and many volunteers provided assistance with fieldwork.

\section{LITERATURE CITED}

Annis SL, Dastoor FP, Ziel H, Daszak P, Longcore JE (2004) A DNA-based assay identifies Batrachochytrium dendrobatidis in amphibians. J Wildl Dis 40:420-428

Aplin K, Kirkpatrick P (1999) Progress report on investigations into chytrid fungal outbreak in Western Australia. Western Australia Museum, Perth

Beard KH, O'Neill EM (2005) Infection of an invasive frog Eleutherodactylus coqui by the chytrid fungus Batrachochytrium dendrobatidis in Hawaii. Biol Conserv 126: 591-595

Berger L, Speare R, Daszak P, Green DE and 10 others (1998) Chytridiomycosis causes amphibian mortality associated with population declines in the rain forests of Australia and Central America. Proc Natl Acad Sci USA 95: 9031-9036

Berger L, Speare R, Hines HB, Marantelli G and 10 others (2004) Effect of season and temperature on mortality in amphibians due to chytridiomycosis. Aust Vet J 82:31-36

Borg I, Rohde G, Loseke S, Bittscheidt J, Schultze-Werninghaus G, Stephan V, Bufe A (2003) Evaluation of a quantitative real-time PCR for the detection of respiratory syncytial virus in pulmonary diseases. Eur Respir J 21:944-951

Bosch J, Martínez-Solano I, García-París M (2001) Evidence of a chytrid fungus infection involved in the decline of the common midwife toad (Alytes obstetricans) in protected areas of central Spain. Biol Conserv 97:331-337

Boyle DG, Boyle DB, Olsen V, Morgan JAT, Hyatt AD (2004) Rapid quantitative detection of chytridiomycosis (Batrachochytrium dendrobatidis) in amphibian samples using real-time Taqman PCR assay. Dis Aquat Org 60:141-148

Burrowes PA, Joglar RL, Green DE (2004) Potential causes for amphibian declines in Puerto Rico. Herpetologica 60: 141-154

Daszak P, Scott DE, Faggioni C, Kilpatrick AM, Gibbons JW, Porter D (2005) Amphibian population declines at Savannah River site are linked to climate, not chytridiomycosis. Ecology 86:3532-3537

Donovan JW, Ladetto M, Zou G, Neuberg D, Poor C, Bowers D, Gribben JG (2000) Immunoglobulin heavy-chain consensus probes for real-time PCR quantification of residual disease in acute lymphoblastic leukemia. Blood 95:2651-2658

Emery SL, Erdman DD, Bowen MD, Newton BR and 12 others (2004) Real-time reverse transcription-polymerase chain reaction assay for SARS-associated coronavirus. Emerg Infect Dis 10:311-316

Ginzinger DG (2002) Gene quantification using real-time quantitative PCR: an emerging technology hits the mainstream. Exp Hematol 30:503-512

Editorial responsibility: Otto Kinne, Oldendorf/Luhe, Germany
Green DE, Converse KA, Schrader AK (2002) Epizootiology of sixty-four amphibian morbidity and mortality events in the USA, 1996-2001. Ann NY Acad Sci 969:323-339

Hanselmann R, Rodrigues A, Lampo M, Fajardo-Ramos L, Aguirre AA, Kilpatrick AM, Rodriguez JP, Daszak P (2004) Presence of an emerging pathogen in introduced bullfrogs Rana catesbeiana in Venezuela. Biol Conserv 120:115-119

Helps C, Reeves N, Tasker S, Harbour D (2001) Use of realtime quantitative PCR to detect Chlamydophila felis infection. J Clin Microbiol 39:2675-2676

Hopkins S, Channing A (2003) Chytrid fungus in Northern and Western Cape frog populations, South Africa. Herpetol Rev 34:4

Kriger KM, Hines HB, Hyatt AD, Boyle DG, Hero JM (2006) Techniques for detecting chytridiomycosis in wild frogs: comparing histology with real-time Taqman PCR. Dis Aquat Org 71:141-148

Lips KR (1999) Mass mortality and population declines of anurans at an upland site in western Panama. Conserv Biol 13:117-125

Luthra R, McBride JA, Cabanillas F, Sarris A (1998) Novel 59 exonuclease-based real-time PCR assay for the detection of $\mathrm{t}(14 ; 18)(\mathrm{q} 32 ; \mathrm{q} 21)$ in patients with follicular lymphoma. Am J Pathol 153:63-68

McDonald KR, Mendez D, Muller R, Freeman AB, Speare R (2005) Decline in the prevalence of chytridiomycosis in frog populations in North Queensland, Australia. Pac Conserv Biol 11:114-120

Okano Y, Hristova KR, Leutenegger CM, Jackson LE, Denison RF, Gebreyesus B, Lebauer D, Scow KM (2004) Application of real-time PCR to study effects of ammonium on population size of ammonia-oxidizing bacteria in soil. Appl Environ Microbiol 70:1008-1016

Ouellet M, Mikaelian I, Pauli BD, Rodrigue J, Green DM (2005) Historical evidence for widespread chytrid infection in North American amphibian populations. Conserv Biol 19:1431-1440

Peccoud J, Jacob C (1996) Theoretical uncertainty of measurements using quantitative polymerase chain reaction. Biophys J 71:101-108

Retallick R, McCallum H, Speare R (2004) Endemic infection of the amphibian chytrid fungus in a frog community postdecline. PLOS Biol 2:1-7

Ron SR, Duellman WE, Coloma LA, Bustamante MR (2003) Population decline of the Jambato toad Atelopus ignescens (Anura, Bufonidae) in the Andes of Ecuador. J Herpetol 37:117-126

Speare R, Skerratt L, Berger L, Hines H and 8 others (2005) A project that designs and trials a pilot survey to map the distribution of chyridomycosis (caused by the amphibian chytrid fungus) in Australian frogs. Final report for project ID 44381 (tender 63/2003) to the Australian Government Department of the Environment and Heritage

Weldon C, du Preez LH (2004) Decline of the Kihansi spray toad, Nectophrynoides asperginis, from the Udzungwa mountains, Tanzania. Froglog 62:2-3

Weldon C, du Preez LH, Muller R, Hyatt AD, Speare R (2004) Origin of the amphibian chytrid fungus. Emerg Infect Dis 10:2100-2105

Submitted: November 15, 2005; Accepted: March 20, 2006

Proofs received from author(s): July 19, 2006 